\title{
SURGERY \\ Benefits of remote ischaemic preconditioning on outcomes after cardiac surgery
}

Intensive remote ischaemic preconditioning (RIPC) can reduce perioperative myocardial injury and the incidence of other postoperative complications. This finding comes from a study performed at the UCLH Heart Hospital, London, UK in 2010-2012.

\section{4 ...this simple, virtually cost-free intervention can improve short-term clinical outcomes... 77}

RIPC - the application of one or several brief cycles of nonlethal ischaemia and reperfusion to a tissue or organ-has been shown to protect the heart against a lethal episode of acute ischaemia-reperfusion injury. However, investigators in some studies have failed to show a reduction in perioperative myocardial injury using a standard single-limb RIPC protocol. Derek Hausenloy and colleagues, therefore, tested the benefits of an intensive RIPC protocol applied simultaneously to the upper arm and thigh.

A total of 180 patients undergoing either CABG or valve surgery were randomly allocated to either RIPC ( $2-5$ min cycles of blood-pressure cuff inflation and deflation applied simultaneously to the arm and leg) or control (cuffs applied to the limbs, but not inflated). Perioperative myocardial injury (assessed at $72 \mathrm{~h}$ after surgery by high-sensitivity assay of the troponin T level; the primary end point of the study) was reduced by $26 \%$ in patients receiving RIPC compared with those in the control group $(P=0.003)$. In the analysis of secondary end points, RIPC was associated with a $54 \%$ reduction in the incidence of postoperative atrial fibrillation $(P=0.031)$, a $48 \%$ reduction in the incidence of acute kidney injury $(P=0.063)$, and a 1-day reduction in the mean duration of stay in the intensive care unit $(P=0.043)$, compared with control.
No significant differences between the two groups were reported in the requirement for inotrope support, or major adverse cardiac events at 6 weeks.

The investigators emphasize that this simple, virtually cost-free intervention can improve short-term clinical outcomes. "To investigate whether all patients undergoing CABG surgery should receive RIPC beforehand, we are investigating in the ERICCA trial ... whether RIPC can improve major adverse cardiac and cerebral events at 1 year," says Dr Hausenloy. "We also plan to investigate the same therapeutic strategy in patients presenting with a ST-segment elevation myocardial infarction treated by primary percutaneous coronary intervention ... in the ERIC-PPCI trial."

Gregory B. Lim

Original article Candilio, L. et al. Effect of remote ischaemic preconditioning on clinical outcomes in patients undergoing cardiac bypass surgery: a randomised controlled clinical trial. Heart doi:10.1136/heartjnl-2014-306178 